\title{
INFLUENCE OF SOLUTION AND AGING TREATMENT CONDITIONS ON THE FORMATION OF ULTRAFINE-GRAINED STRUCTURE OF CuFe2 ALLOY PROCESSED BY ROLLING WITH CYCLIC MOVEMENT OF ROLLS
}

\begin{abstract}
The effect of second phase particles on grain refinement in $\mathrm{CuFe} 2$ alloy has been investigated by using rolling with the cyclic movement of rolls (RCMR) method. Two different population of second phase particles of Fe: coherent, about $10 \mathrm{~nm}$ in diameter and about $100 \mathrm{~nm}$ in size were obtained by applying aging treatment followed at $500^{\circ} \mathrm{C}$ for $2 \mathrm{~h}$ and at $700^{\circ} \mathrm{C}$ for $24 \mathrm{~h}$ respectively. In addition, solution treated samples were deformed by RCMR method at the same parameters. The microstructures of the $\mathrm{CuFe} 2$ alloy were analyzed using light microscope (LM), electron backscattered diffraction (EBSD) microscope technique and scanning transmission electron microscope (STEM). The presence of high-density of coherent Fe particles in the matrix inhibits recovery process and in the result obtained grain/subgrain boundaries have diffused character and are weakly visible. The largest particles which are not coherent with the matrix act as an effective barrier against the boundary motion.
\end{abstract}

Keywords: CuFe2 alloy, severe plastic deformation, ultrafine-grains, EBSD, STEM

\section{Introduction}

Severe plastic deformation (SPD) techniques are widely applied for production of ultrafine-grained (100-1000 nm in size) and nano-grained (less than $100 \mathrm{~nm}$ in size) microstructures in bulk materials [1-4]. Materials produced by SPD have good mechanical and physical properties [5-8]. Age- hardening effect in connection with grains refinement to ultrafine scale are the main approaches to improve strength and ductility. One of the methods which can be used for SPD processing is rolling with the cyclic movements of rolls (RCMR) because it allows for large deformations, and therefore grain refinement is possible [9]. This original method of deformation has been patented at Silesian University of Technology, Faculty of Materials Engineering and Metallurgy in Poland [10]. The basis of this method is deformation of a massive sample by simultaneously applying rolling and transverse to the rolling direction - cyclic movements of rolls. The value of the total effective strain $\mathrm{e}_{\mathrm{ft}}$ can be controlled by changing the proportions of the following parameters: rolling reduction $\varepsilon_{h}(\%)$, rolling rate $v(\mathrm{rpm})$, amplitude of transverse movement of rolls $A(\mathrm{~mm})$, frequency of transverse movement of rolls $f(\mathrm{~Hz})$. Application of cyclic deformation mode (RCMR belongs to this method) to grain refinement introduces some restrictions in structure refinement. Obtained in literature $[6-8,13]$ results suggest that the recovery process plays a significant role in formation of the ultrafine-grained structure. These structural effects such as the transformation of dislocation tangles into polygonal boundaries and annihilation of dislocations, may have a negative influence on the grain refinement. The decrease in dislocation density doesn't lead to the formation of an ultrafine-grained structure. Only large quantity of dislocations in grain boundaries favorably become effective barriers to suppress recovery processes. Pronounced recovery during deformation results in decrease of mechanical properties. This phenomenon occurs especially in pure $\mathrm{Cu}[11,12]$.

It is important to know whether the similar mechanism of microstructure recovery is effective in the precipitation hardened materials. It is well-known that the presence of second phase particles could potentially have a significant effect on the grain refinement [13-17]. No investigations have been performed however on the effect of second phase precipitates on grain refinement during ( $\mathrm{RCMR}$ ) in $\mathrm{CuFe} 2$ alloy. For this reason, in the present work, effect of second phase precipitates on the deformed microstructure by rolling with cyclic movement of rolls was studied. Obtained particles during application of various parameters of heat treatments are different in dimension, distance between them and crystal lattice fit.

\section{Materials and experimental procedures}

In this study, precipitation hardened copper alloy with addition of $2 \%$ Fe was used. This alloy was selected for investigation because can be readily produced by SPD processing at room temperature. The alloy was prepared by melting and alloying in open-air induction furnace, followed by casting into the mould with diameter of $50 \mathrm{~mm}$. Ingots were hot rolled into

\footnotetext{
* SILESIAN UNIVERSITY OF TECHNOLOGY, FACULTY OF MATERIALS ENGINEERING AND METALLURGY, 8 KRASINSSIEGO STR., 40-019 KATOWICE, POLAND

** INSTITUTE FOR FERROUS METALLURGY, 12-14 K. MIARKI STR., 44-100 GLIWICE, POLAND

* Corresponding author: anna.urbanczyk-gucwa@polsl.pl
} 
bars (Fig. 1a) with dimensions of $8 \times 8 \times 60 \mathrm{~mm}$. The samples were prepared through:

1) solution treatment at $1000^{\circ} \mathrm{C}$ for $3 \mathrm{~h}$ followed by quenching into iced water (in this article referred to as " $\mathrm{S}$ " state)

2) solution and aging treatment at $500^{\circ} \mathrm{C}$ for $2 \mathrm{~h}$ (referred to as "S+1A")

3) solution and aging treatment at $700^{\circ} \mathrm{C}$ for $24 \mathrm{~h}$ (referred to as "S+2A")

Specimens with three distinctly different initial conditions were deformed by using RCMR method. In our experiments, $\varepsilon_{h}=80 \%, v=0.7 \mathrm{rpm}, \mathrm{A}=0.8 \mathrm{~mm}, f=1.5 \mathrm{~Hz}$. Process was realized in 6 passes. The total effective strain $\varepsilon_{f t}$ was $\sim 5$. The RCMR method and formula of total effective strain calculation has been described in more details in [9].

The examples of samples after deformation are presented in Figure $1 \mathrm{a}$ ) and b). All tests by using the RCMR method were conducted at room temperature. The samples in solution condition and next deformed by RCMR are referred to as "S+RCMR" and samples in solution and aging state and next deformed by $\mathrm{RCMR}$ are referred to as " $\mathrm{S}+1 \mathrm{~A}+\mathrm{RCMR}$ " and " $\mathrm{S}+2 \mathrm{~A}+\mathrm{RCMR}$ ".

The investigation of the ultrafine-grained structures was performed using a FEI INSPECT F scanning electron microscope
(SEM) equipped with a cold field emission gun and an electron backscatter diffraction (EBSD) detector. SEM specimens were polished by using an ion thinning device (PECS) manufactured by Gatan, Inc. The polishing was performed using the following parameters: beam current $-2,5 \mathrm{keV}$, and total time $-70 \mathrm{~min}$. During the process the sample was rotating with a rate of $15 \mathrm{rpm}$. Moreover, tilt angle was applied. It was changing with a range of $0-20^{\circ}$ with $8^{\circ} / \mathrm{sec}$ for $45 \mathrm{~min}$, and then the tilt angle was decreased to $0-10^{\circ}$. Additionally, scanning transmission electron microscope (STEM) Hitachi HD-2300A was used for microstructure characterization. The mechanical properties of the alloys were characterized by hardness measurements. The Vickers hardness was measured on an electrolytically polished surface of the samples by means of Future-Tech. Corp. Fm-800. A $1000 \mathrm{~g}$ load applied for $15 \mathrm{~s}$ was ensured for these measurements. The hardness values were taken as the average of a minimum of 10 measurements. Due to heterogeneity of the deformation, the SEM/EBSD observations and hardness measurements were made in areas located at a distance of about $1 / 4$ of the sample's height in the transverse plane section. STEM observations of the thin foils parallel to the rolling plane were performed from distance of about $1 / 4$ of the sample's height (Fig. 1d).
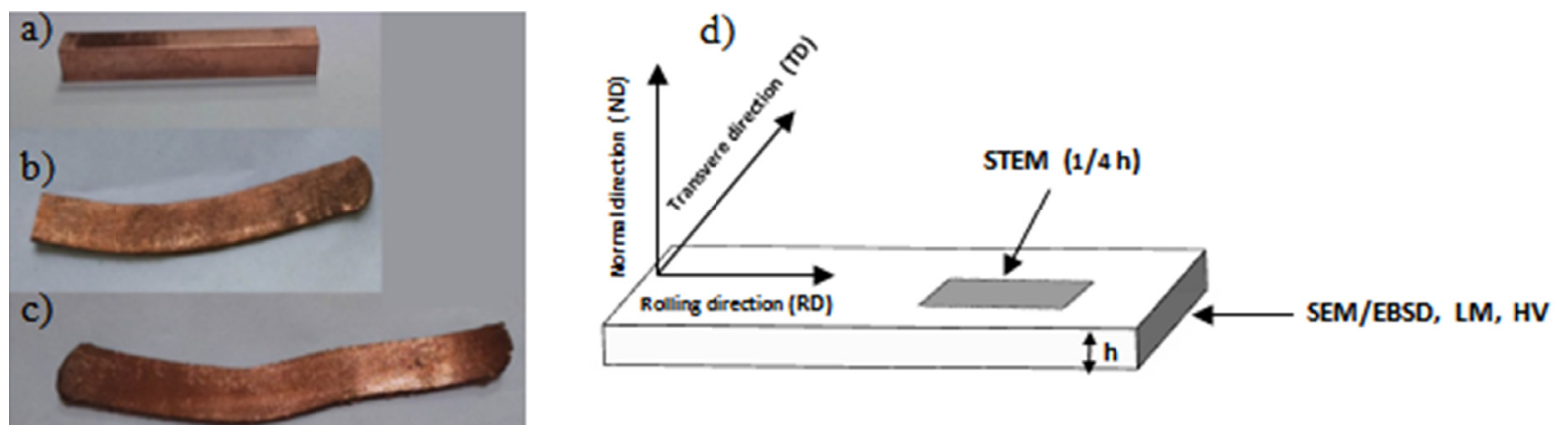

Fig. 1. Macrostructure of $\mathrm{CuFe} 2$ alloy, initial sample before deformation a) and samples after $\mathrm{S}+1 \mathrm{~A}+\mathrm{RCMR}$ and $\mathrm{S}+2 \mathrm{~A}+\mathrm{RCMR}$ processing b) and c) position where specimens for microstructure analysis and hardness measurements were taken from the deformed specimens $\mathrm{d}$ )

\section{Results and discussion}

Figure 2a shows the microstructure of $\mathrm{CuFe} 2$ alloy after solution treatment. Microstructure is characterized by the presence of undissolved equiaxial $\mathrm{Fe}$ precipitates in $\mathrm{Cu}$ matrix. Based on STEM/EDS investigations it has been shown, that the precipitates are rich in P. Phosphorus was added as an antioxidant during casting. The average diameter of precipitates is about $0.5 \mu \mathrm{m}$ and average distance of particles is about $4.5 \mu \mathrm{m}$ (Fig. 2b,c). The initial grain size of the specimen in the solution treatment conditions was determined as $200 \mu \mathrm{m}$.
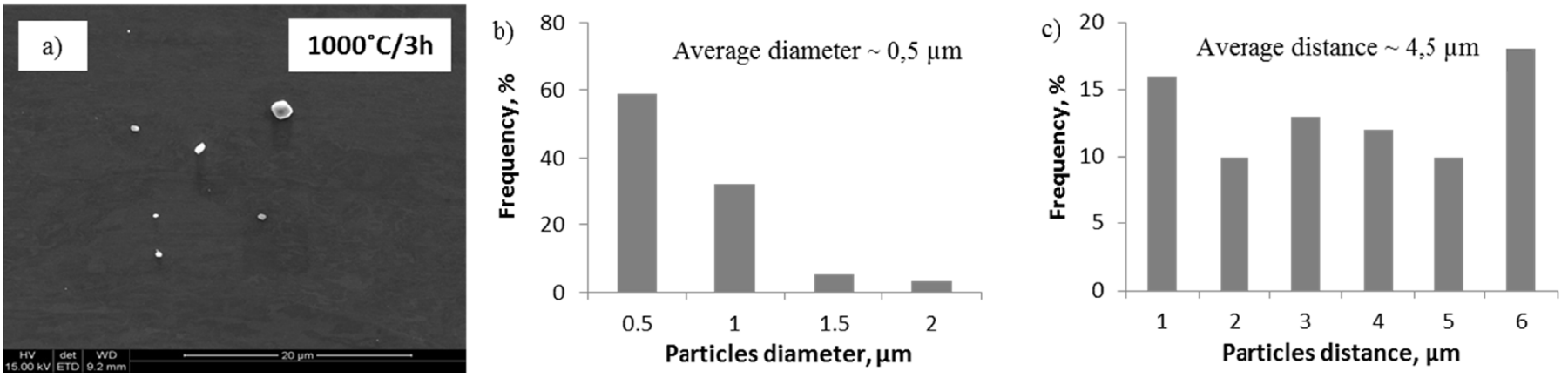

Fig. 2. Microstructure of $\mathrm{CuFe} 2$ alloy after solution treatment a), distribution of Fe particles b) and distribution of Fe particles distance c) 
Fig. 3 and Fig. 4 show the microstructure of $\mathrm{CuFe} 2$ alloy after solution and aging treatment followed at $500^{\circ} \mathrm{C}$ for $2 \mathrm{~h}$ and at $700^{\circ} \mathrm{C}$ for $24 \mathrm{~h}$. Aging treatment at $500^{\circ} \mathrm{C}$ for $2 \mathrm{~h}$ results in the formation of coherent $\mathrm{Fe}$ precipitates within the matrix (Fig. 3a). The size of these particles varies between 10-25 nm
(Fig. 3b). With prolonged aging temperature and time, the precipitates lost coherence with matrix (Fig. 4a) and amount of the particles within the matrix decreases (spacing between particles increase). The size of these particles varies between 60 to $140 \mathrm{~nm}$ (Fig. 4b).
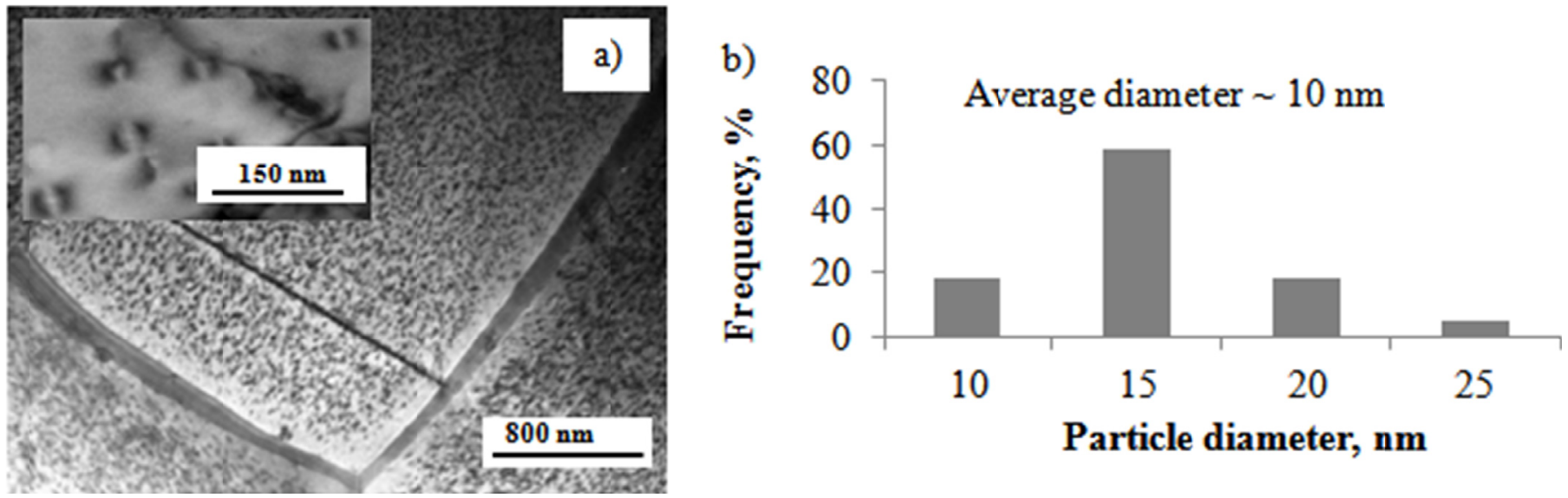

Fig. 3. Microstructure of $\mathrm{CuFe} 2$ alloy after ageing in $500^{\circ} \mathrm{C} / 2 \mathrm{~h}$ a) and distribution of average diameter of coherent Fe precipitates b)
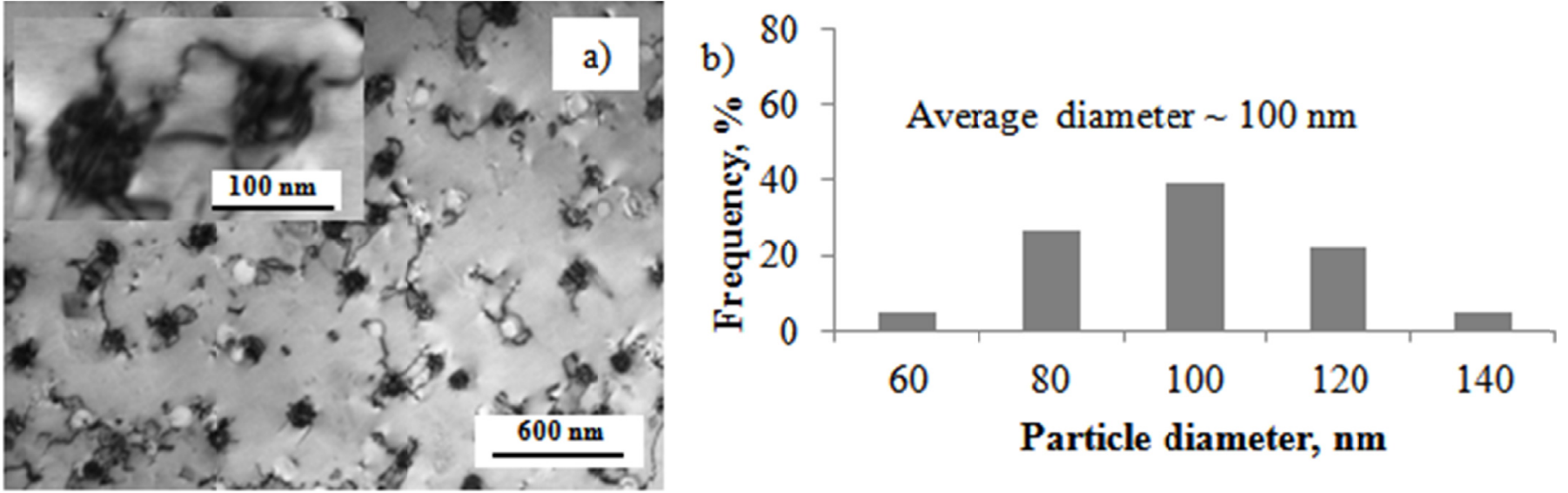

Fig. 4. Microstructure of $\mathrm{CuFe} 2$ alloy after ageing in $700^{\circ} \mathrm{C} / 24 \mathrm{~h}$ a) and distribution of average diameter of noncoherent Fe precipitates b)

Figures 5-7 show boundary misorientation maps constructed from EBSD results. In the misorientation maps, the thin lines represent the boundaries having smaller misorientation $\theta$ in the range of $2 \leq \theta<15^{\circ}$, while the bold lines represent the boundaries with larger misorientation of $15 \leq \theta$. Low angle boundaries with misorientation smaller than $2^{\circ}$ were out of consideration. Figures show that all microstructures processed by RCMR method have low and high angle boundary structures elongated along RD.
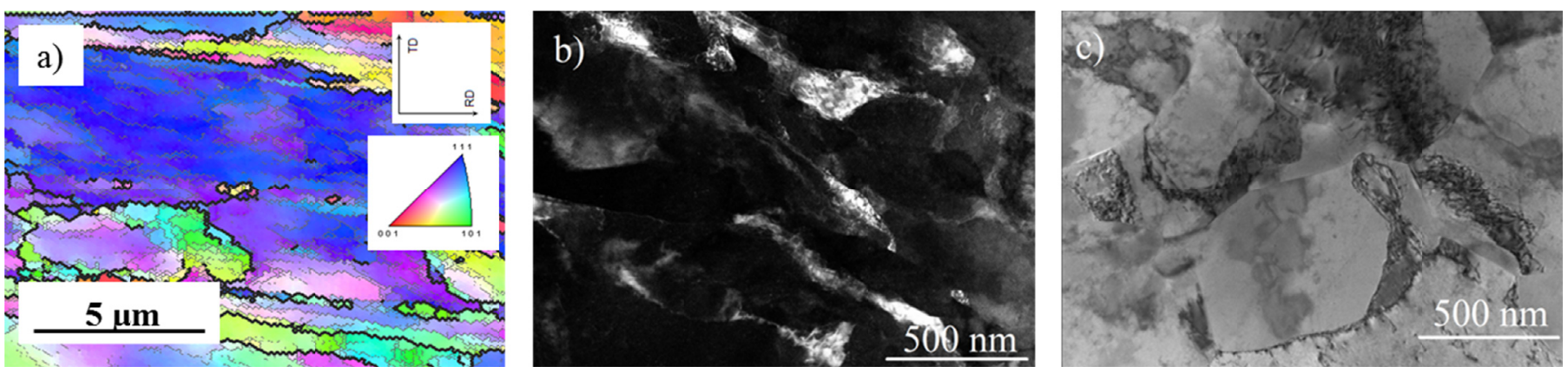

Fig. 5. EBSD map a) and STEM micrographs in dark - field image b) and bright- field image, typical for S+RCMR state 

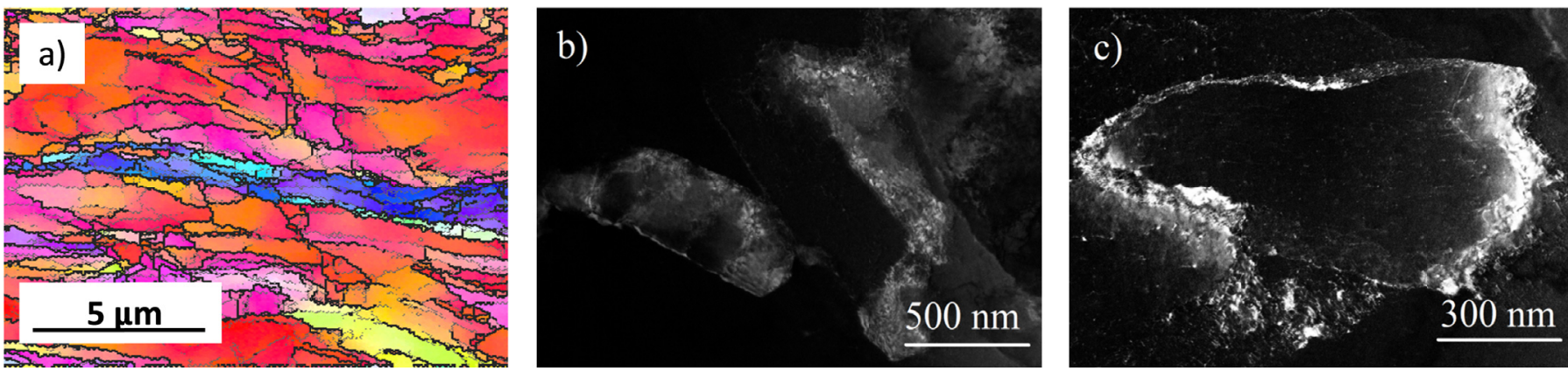

Fig. 6. EBSD map a) and STEM micrographs in dark-field image b, c) typical for S+1A+RCMR state
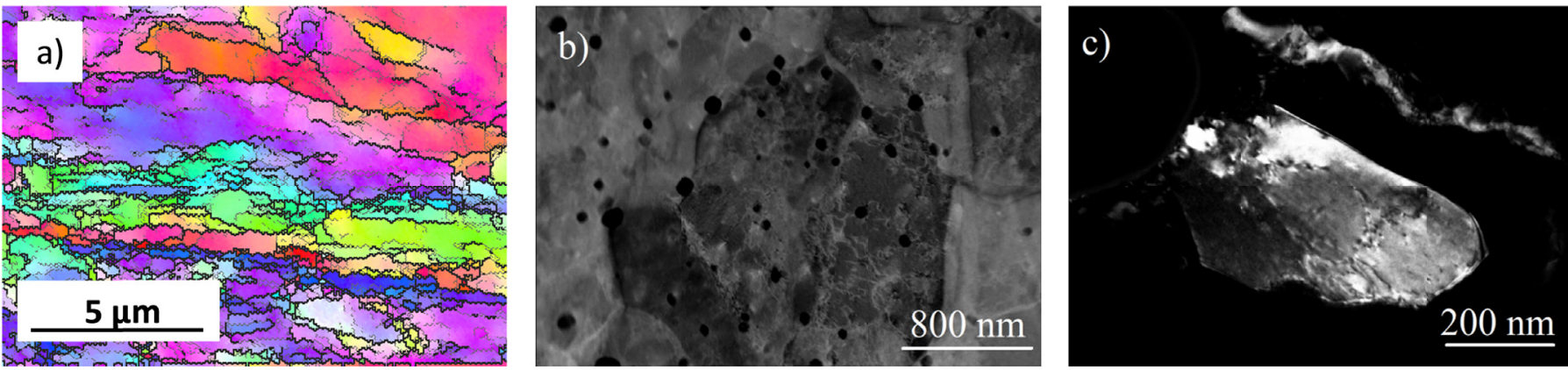

Fig.7. EBSD map a) and STEM micrographs in dark- field image b, c) typical for S+2A+RCMR state

The map in S+RCMR state obtained using the EBSD technique is shown in Figure 5a. The microstructure is characterized by boundaries with small and high misorientation angles. Visible fragments of high angle boundaries (HABs) belong to the original grain boundaries and the shape of the primary grains is related to the direction of the RCMR deformation. Only individual equiaxal ultrafine-grains of the HABs are visible. From the EBSD data, the fraction of HABs is determined and reached about $23 \%$ as is plotted in Figure 8 . The average grain/subgrain size was $0.49 \mu \mathrm{m}$ and $0.42 \mu \mathrm{m}$ respectively.

The refined microstructure is characterized by a mixture of equiaxed and elongated subgrains/grains with dislocations (Fig. 5b,c). Very often equiaxed subgrains/grains are near without of dislocation inside (Fig. 5c). Many of the visible boundaries are straight, however very often curved boundaries are observed. This means that motion of grains is possible. $\mathrm{S}+1 \mathrm{~A}+\mathrm{RCMR}$ specimen shows the ultrafine lamellar structure with HABs elongated to RD but very often the structure is subdivided by other lamellar structures (Fig. 6a). Intersection of ultrafine lamellar bands is typical feature of microstructure for $\mathrm{S}+1 \mathrm{~A}+\mathrm{RCMR}$ state. The STEM micrograph (Fig. 6b) shows the tangles of dislocations and elongated subgrains (dense dislocation walls)/grains with high dislocation density. In some microareas, the boundaries of new grains/subgrains are poorly defined (Fig. 6c). The fraction of HABs is lower than that of the S+RCMR state (Fig. 8). It should be noted that for $\mathrm{S}+1 \mathrm{~A}+\mathrm{RCMR}$ state relatively large amount of MABs (medium angle boundary) is observed. Finally, fractions HABs of $20 \%$ and MABs of $40 \%$ were achieved. The presence of coherent precipitates especially reduces the mobility of dislocations and as an effect, dense dislocation walls slowly develop into the grain boundaries. From EBSD measurement the average grain/subgrain size was determined as $0.39 \mu \mathrm{m}$ and $0.36 \mu \mathrm{m}$ respectively.

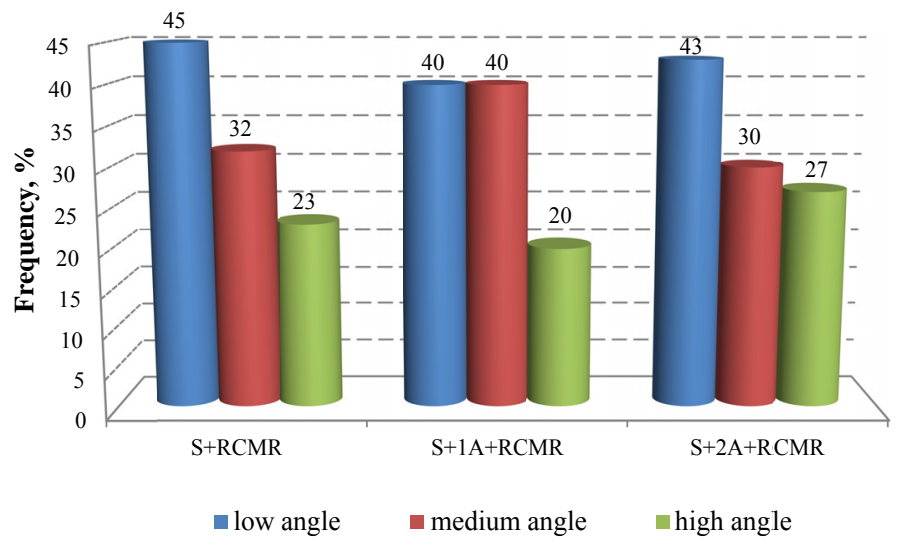

Fig. 8. Changes of misorientation angles in dependence of deformation state. The misorientation angles have been divided into three ranges: low angle $-2^{\circ}-5^{\circ}$, medium angle - above $5^{\circ}-15^{\circ}$, high angle above $15^{\circ}$

On EBSD map obtained for $\mathrm{S}+2 \mathrm{~A}+\mathrm{RCMR}$ state there are some well visible ultrafine equiaxed grains/subgrains with random orientations (Fig. 7a). In S+2A+RCMR state a structure of equiaxed grains with dislocations inside or without dislocations, which are quite frequently delineated by sharp boundaries was developed (Fig. 7 b-c). The fraction of HABs is $27 \%$ (Fig. 8). The average grain/subgrain size was determined as $0.44 \mu \mathrm{m}$ and $0.40 \mu \mathrm{m}$ respectively. Typical examples of deformed structure with small Fe particles are shown in Figure 7b. The precipitates 
are located in the grain/subgrain boundaries and limit the grain growth by providing local pinning of the grain boundary. Additionally precipitates resided inside grains/subgrains. In this case, high density of dislocation - was observed around most of Fe particles.

Figure 9 shows Vickers hardness of the $\mathrm{CuFe} 2$ alloy for different conditions of heat treatment and RCMR deformation. The specimens after ageing $(\mathrm{S}+1 \mathrm{~A}$ and $\mathrm{S}+2 \mathrm{~A})$ showed much higher hardness than the $\mathrm{S}$ state. This was due to the presence of precipitates in the aged specimens. Higher values of hardness observed in the sample aged at $500^{\circ} \mathrm{C} / 2 \mathrm{~h}$ are attributed to the formation of very small particles which are coherent with the matrix. The increase in hardness for samples in S+RCMR state compared with $\mathrm{S}$ state should be attributed to the strong deformation and generation of dislocation. The best results in hardness measurement (150 HV1 and $133 \mathrm{HV} 1)$ were obtained for all alloys in $\mathrm{S}+1 \mathrm{~A}+\mathrm{RCMR}$ and $\mathrm{S}+2 \mathrm{~A}+\mathrm{RCMR}$ states. The enhancement of hardness in $\mathrm{S}+1 \mathrm{~A}+\mathrm{RCMR}$ state may be attributed both the ultrafine-grained matrix with high dislocation density and the presence of fine precipitates which provide resistance to dislocation slip and increased dislocation accumulation. The hardness measurements were approximately two-times higher after deformation than in the initial state. The alloy was deformed for a total effective strain $\varepsilon_{f t} \sim 5$. The sample in S+RCMR state exhibit a typical large strained structure, i.e. subgrains/grains with high dislocation density in the interior and with mixture of low angle and high angle boundaries (Fig. 5). This means that it is difficult to obtain an ultrafine-grained structure with high fraction of HABs under RCMR deformation. Reversible cyclic methods of deformation delay process of dislocations rearrangement, which run on the same slip planes. For this reason, the cyclic extrusion compression (CEC) method is slower than the hydrostatic extrusion (HE) and as equal-channel angular pressing (ECAP) methods [18]. The RCMR method is cyclic, and thus high values of total effective strain must be used for grain refinement with high fraction of HABs. In S+1A+RCMR state the presence of dislocations inside new grains/subgrains and inside grains/ subgrains boundaries $(\mathrm{S}+1 \mathrm{~A}+\mathrm{RCMR}$ state appears to be most efficient in formation of non-equilibrium grain boundaries) should be attributed to the strong deformation and mainly by increase the rate of dislocation generation by encouraging the formation of Orovan loops. It should be taken into account that the high amount of dislocation produced in the first passes of RCMR thanks to presence of fine precipitations can strongly accelerate formation of numerous band deformations. Barlow et al. [19] have reported that dispersed particles are effective in reduction of the slip distances. In the result the lamellar structure with finer spacing is thus retained during deformation. During next passes, the coherent precipitates inhibit the long-range migration of dislocation and, less dynamic boundary migration occurs. Therefore, it is possible to obtain much finer deformation of structures. This result suggests that in order to improve the strength (hardness) by RCMR it is recommended to process $\mathrm{CuFe} 2$ alloy at $\mathrm{S}+1 \mathrm{~A}+\mathrm{RCMR}$ state.

In $\mathrm{S}+2 \mathrm{~A}+\mathrm{RCMR}$ state, the grain/subgrain boundaries are strongly pinned by fine precipitates with $100 \mathrm{~nm}$ dimension. In

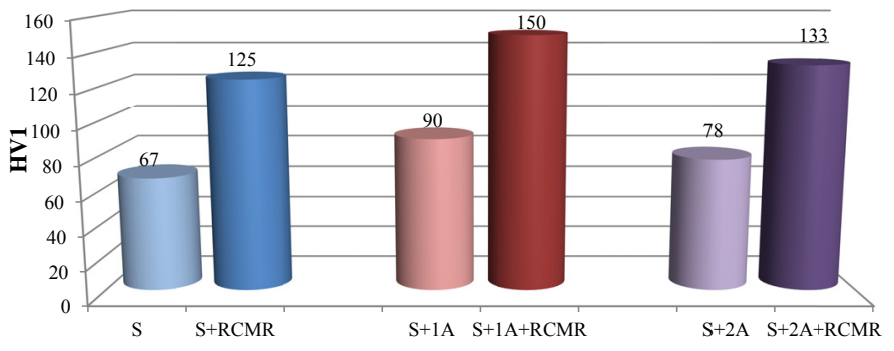

Fig. 9. Changes in hardness of $\mathrm{CuFe} 2$ alloy after heat treatment and heat treatment + RCMR

the result the structure recovery is suppressed in large extent because these particles constitute additional barriers to the movement of grains/subgrains. STEM research has shown, that these precipitates stopped not only motion of grains/sugrains but also partly stopped dislocation motion inside grains/subgrains, because in favorable conditions i.e. when spaces between them are small, the Fe precipitates were enclosed by dislocation network.

In literature the effect of influence of fine particles on grain refinement in $\mathrm{CuFe} 2$ alloy is not well documented. Research carried out in $\mathrm{CuFe} 2$ alloy after solution and rolling with $50 \%$ reduction, shown that the hardness was 155 HV5 [20]. After applying ECAP methods with a strain of 8 for deformation with route $\mathrm{Bc}$ (in $\mathrm{Bc}$ route the specimen is rotated $90^{\circ}$ counter-clockwise around its longitudinal axis after each pass) for $\mathrm{Cu}-\mathrm{Fe}-\mathrm{P}$ alloy in the as-cast state, an ultrafine-grain structure consisted of grains and subgrains was produced and the grain were nearly equiaxed, ranging from $50 \mathrm{~nm}$ to slightly larger than $200 \mathrm{~nm}$ [14]. In as-cast state, the microhardness was $80 \mathrm{HV} 0.1$ and after SPD deformation was $167 \mathrm{HV} 0.1$. Our results are comparable with other methods of SPD.

\section{Conclusions}

Precipitation-hardened $\mathrm{CuFe} 2$ alloy was solution-treated and aged to prepare different microstructures, which may have quite different effect on the grain growth during SPD. SPD process was realized by using RCMR method. The main results obtained in this experiment are as follows:

The transverse movement of rolls refined structure of $\mathrm{CuFe} 2$ alloy. The mean grain/subgrain size in S+RCMR state was 0.49 $\mu \mathrm{m}$ and $0.42 \mu \mathrm{m}$ respectively. The fraction of high angle grain boundaries was $23 \%$ but the fraction of medium angle grain boundaries was $32 \%$. Processes of recovery appeared after subjecting of the samples to a total effective strain $\varepsilon_{f t} \sim 5$. Presence of second-phase particles have a significant effect on formation of ultrafine-grained structure during RCMR deformation:

- Existence of fine coherent particles (10 $\mathrm{nm}$ in size) is effective in dislocations pinning and increase of dislocations density. Inside new grains/subgrains and inside grain/subgrain boundaries high amount of dislocations are present.

- The particles with the average dimension of $100 \mathrm{~nm}$ were found to be effective in hindering dislocation and grain/ subgrain boundaries motion. 
Due to the nature of the RCMR method, promising state in grain refinement effect is $\mathrm{S}+1 \mathrm{~A}+\mathrm{RCMR}$. The specimens showed ultrafine-grained structure with grain/ subgrain size about $0.39 \mu \mathrm{m}$ and $0.36 \mu \mathrm{m}$ respectively. The fraction of high angle grain boundaries was $20 \%$ but the fraction of medium angle grain boundaries was $40 \%$.

\section{Acknowledgments}

This work was carried out with project No. UMO-2013/09/B/ST8/01695

\section{REFERENCES}

[1] H. Gleiter, Acta Mater. 48, 1-29 (2000).

[2] S.C. Tjong, H. Chen, Mater. Sci. Eng. A 45, 1-88 (2004).

[3] R.Z. Valiev, Nan. Tech. for Mil. Veh. 1-12 (2006).

[4] R.Z. Valiev, R.K. Islangaliev, I.V. Aleksandrov, Prog. in Mat. Sci. 45, 103-189 (2000).

[5] Valiev R.Z, et al., Dok. Phy. 46, 633-635 (2001).

[6] Wang Y.M. et al., Acta Mater. 52, 1699-1709 (2004).
[7] W. Bochniak, K. Marszowski, A. Korbel, J. of Mat. Pro. Tech. 169, 44-53 (2005).

[8] P.B. Prangnell, J.R. Boven, A. Gholinia, Mat. Sci. 105-126 (2001).

[9] Z. Cyganek, K. Rodak, F. Grosman, Arch. Civ. Mech. Eng. 13, 7-13 (2013).

[10] Patent No. PL 203220 B1.

[11] K. Rodak, R. Molak, Z. Pakiela, Phy. Sta. Sol. 7, 1351-1354 (2010).

[12] K. Rodak, G. Moskal, Key Eng. Mat. 465, 330-333 (2010).

[13] P.J. Apps, M. Berta, P.B. Prangnell, Acta Mat. 53, 499-511 (2005).

[14] H. Cao, J.Y. Min, S. D. Wu, A. P. Xian, J.K. Shang, Mat. Sci. and Eng. A 431, 86-91 (2006).

[15] E. Borhani, H. Jafarian, J. of Ult. Gra. and Nano. Mat. 47, 1-7 (2014).

[16] W. Głuchowski, J.P. Stobrawa, Z.M. Rdzawski, Arch. Mat. Sci. and Eng. 47, 103-109 (2011).

[17] A. Gholina, P.B. Prangnell, M.V. Markushev, Acta Mater. 48, 1115-1130 (2000).

[18] P.L. Sun, Scr. Mat. 47, 377-381 (2002).

[19] C.Y. Barlow, N. Hansen, Y.L. Liu, Acta Mater. 50, 171 (2002).

[20] Z. Rdzawski, J. Stobrawa, W. Głuchowski, Arch. Mat. Sci. and Eng. 33, 7-18 (2009). 